\title{
Revealing the initial reaction behavior in the continuous synthesis of metal-organic frameworks using real-time synchrotron $\mathrm{X}$-ray analy- sis
}

\author{
Angelos Polyzoidis, ${ }^{1, *}$ Martin Etter, ${ }^{2}$ Michael Herrmann, ${ }^{1}$ Stefan Loebbecke ${ }^{1}$ and Robert. E. Dinnebier ${ }^{3}$ \\ ${ }^{1}$ Fraunhofer Institute for Chemical Technology ICT, Pfinztal, 76327, Germany. \\ ${ }^{2}$ Deutsches Elektronen-Synchrotron (DESY), Hamburg, 22607, Germany. \\ ${ }^{3}$ Max Planck Institute for Solid State Research, Stuttgart, 70569, Germany. \\ Supporting Information Placeholder
}

\begin{abstract}
In recent years, continuous process technologies have attracted increasing attention, as a means of overcoming limitations in the repeatability and reproducibility of metal organic frameworks (MOFs) synthesis. Research in this area, however, has neglected to provide insight into the phenomena leading to the formation of MOFs. In this work, we report the adaption of highenergy synchrotron X-ray powder diffraction analysis to a continuous ZIF-8 production process for the on-line monitoring of the reaction behavior and crystallite formation during the first seconds of MOF synthesis. It was confirmed that a diffusioncontrolled growth mechanism was accelerated by additional radial diffusion processes in the flow reactor. Kinetic analysis revealed a rapid crystallite formation of ZIF-8 which was completed after 8 seconds of reaction time, and which offers potential for future process optimization.
\end{abstract}

Over the past twenty years, world-wide research on metalorganic frameworks (MOFs) has produced an impressive amount of results, ranging from fundamental findings to technical applications of MOFs. ${ }^{1,2}$ The synthesis of these materials has traditionally been dominated by batch processes. ${ }^{3}$ However, over the last few years an increasing number of publications have demonstrated the benefits of continuous MOF syntheses. ${ }^{4,5}$ It has become evident that continuous MOF processes are able to produce the desired products much faster than batch processes under similar reaction conditions, thus providing significantly enhanced space-time yields.

However, a particular understanding of the MOF formation processes that are relevant under continuous conditions is missing. In order to further enhance the performance of these materials, a deeper understanding of their initial formation and crystal growth processes is crucial. In-situ diffractometric techniques play an essential role in revealing this information.

Numerous techniques for determining the crystal growth mechanisms and kinetics of various MOFs have been implemented ex-situ almost parallel to their invention. ${ }^{6-8}$ In-situ studies concerning the crystallization of MOFs were first introduced nearly a decade later ${ }^{9}$ and since then, several MOFs ${ }^{10-14}$ as well as synthetic processes ${ }^{15}$ have been monitored on-line. Nonetheless, these methods have not yet been applied to the continuous synthesis of MOFs. To the best of our knowledge, this work is the first available report of an insitu monitoring of a continuous MOF synthesis.

The high chemical and thermal stability, wide applicability and convenient synthetic methods make ZIF-8 (zeolitic imidazolate framework-8) one of the most investigated MOFs. The continuous production of this MOF was followed using high resolution, high energy synchrotron X-ray powder diffraction. The experiments were conducted with the aim of optimizing the process with respect to reaction time, and understanding the crystallization kinetics and crystal formation process during the first seconds of the reaction.

The synthesis of ZIF-8 was conducted by mixing an aqueous ammonia solution of $\mathrm{Zn}\left(\mathrm{NO}_{3}\right)_{2}$ with a methanol solution of 2-methyl-imidazole in a T-type micromixer installed at the entrance of a wound tube reactor. ${ }^{16}$ This tailored flow reactor was adapted to the Petra III synchrotron beamline P02.1 at line at DESY (Hamburg, Germany) using a movable stage to focus the synchrotron beam successively on different reactor positions along the flow direction. A detailed description of the experimental process, together with some pictures of the setup, can be found in the supporting information.

Figure 1 depicts the progression of the reaction through a waterfall plot of the acquired XRPD patterns. The bottom pattern represents the first measurement at the entrance of the reactor and corresponds to a reaction time of $3 \mathrm{sec}$. Every following pattern was taken at the subsequent reactor position, corresponding to a reaction time of $+5 \mathrm{sec}$ after the previously taken XRPD pattern.

All patterns are dominated by a strong diffraction peak at $2.4^{\circ} 2 \theta$ and two halos around 2.2 and $5.2^{\circ} 2 \theta$. These signals represent the reflections of the reaction tube, as revealed by a blank measurement conducted prior to the main experiments. Additionally, small peaks (marked with arrows) of increasing intensity, which can be identified as reflections of the ZIF-8 product, ${ }^{17,18}$ emerge in various parts of the diffractogram, 
representing the formation of ZIF-8 and confirming the capability of the in-situ monitoring of the continuous process.

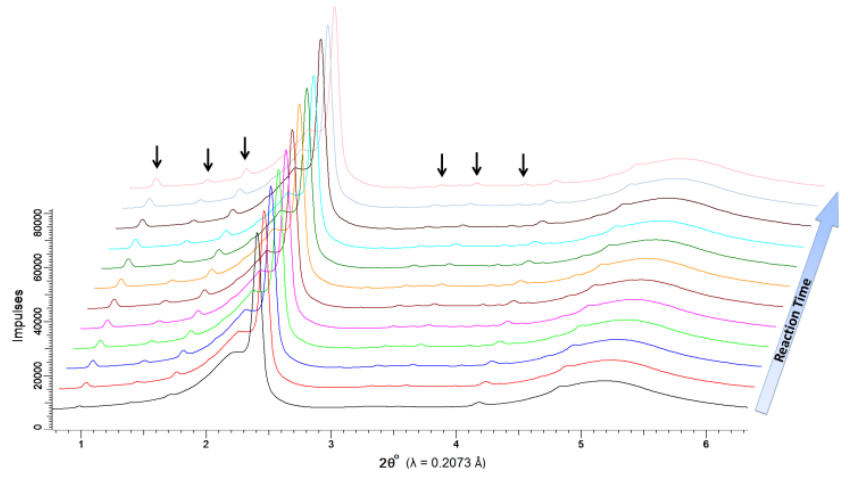

Figure 1: Waterfall plot of the XRPD patterns during ZIF-8 formation measured along the reactor tube (arrows mark ZIF8 reflections).

The raw diffraction patterns were evaluated using the fit and pattern decomposition tools of the program TOPAS 4.2 (Bruker AXS) as well as crystal structure and reference data of ZIF-8. ${ }^{17,18}$ The areas under peaks and halos were quantified using analytical pseudo Voigt profiles. Figure 2, for instance, shows the decomposition of one of the diffraction patterns (red line), and the extracted intensities of the ZIF-8 peaks (blue line).

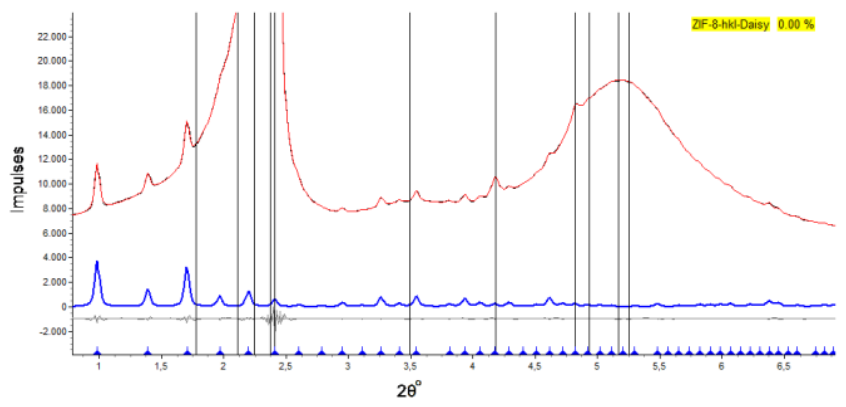

Figure 2: Raw pattern decomposition (red) and extracted ZIF-8 profile (blue).

Based on this data analysis, any formation of intermediate crystalline phases during the ZIF- 8 crystal formation process can be undeniably excluded. In-situ pair distribution function (PDF) analysis could provide additional information concerning the formation mechanism. ${ }^{19}$ The decomposition of the patterns also yielded absolute and relative intensities of the ZIF-8 peaks and the reflections from the reaction tube. Relative intensities may be used to level out intensity fluctuations e.g. of the X-ray source, but may also be prone to a changing thickness of the reaction tube, or if the measuring position shifts out of the tube center. The relative intensities from the diffraction peaks between 0.8 and $4.8^{\circ} 2 \theta$ that belong to the crystal structure of ZIF- 8 evaluated as hkl-phase, in relation to the reaction time are shown in Figure 3 and were used for a kinetic evaluation of the initial ZIF-8 crystallization process. Intensities of the peaks (031), (222), (321) between 2.1 and $2.5^{\circ} \theta$ and (006) at $4.2^{\circ} \theta$ were excluded due to significant overlap with diffraction intensities from the tube and/or sample holder.

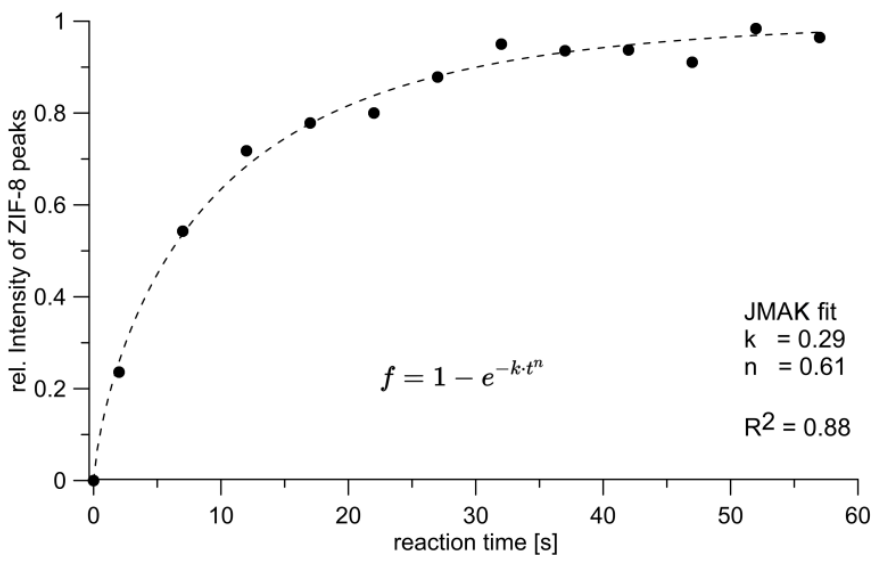

Figure 3: Relative intensity of the ZIF-8 peaks plotted against reaction time, and kinetic evaluation using a JMAK fit.

A fitting of the Johnson-Mehl-Avrami-Kolmogorov (JMAK) equation ${ }^{20-23}$ to the experimental data was compiled, allowing calculation of the temperature-independent Avrami exponent of $\mathrm{n}=0.61(3)$, indicating a diffusion-controlled growth mechanism. ${ }^{24}$

Such a mechanism was expected as a result of the laminar flow conditions present in the applied reactor. However, the small cross section of the reactor tube (approx. 1,600 $\mu \mathrm{m}$ ) causes a significant contribution of radial diffusion processes in addition to the axial diffusion in the flow direction, constraining the mixing of the reactants to be conducted in a diffusion-controlled but nonetheless efficient manner.

Further information was extracted from the XRPD data by means of size/strain analysis which is implemented in the TOPAS program. Based on the geometric peak profiles calibration using the standard $\mathrm{LaB}_{6}$ for reference measurements $^{25}$, the Lorenzian crystallite size parameter was estimated. The analysis was performed applying an hkl-phase for ZIF-8 which constrains the positions and the microstructural parameters of the peaks while keeping the strain broadening unrefined.

Figure 4 depicts the changes to the Lorentzian crystallite size parameter as a function of reaction time. The diagram demonstrates a rapid increase in the average size of coherently scattering domains to about $110 \mathrm{~nm}$ during the first 8 seconds of the reaction, followed by essentially no significant variation as the reaction time progresses. 


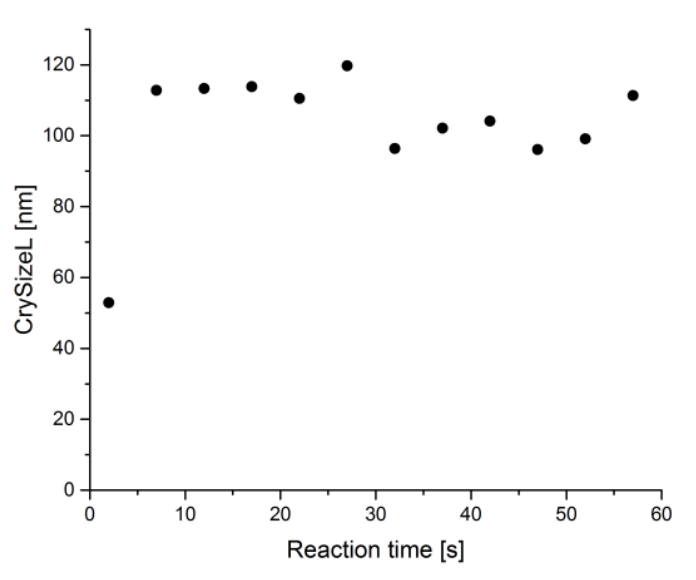

Figure 4: Lorentzian crystallite size of ZIF-8 plotted against reaction time.

This observation can be explained by the fact that the reaction reaches a stationary state after only 8 seconds, and crystal growth can no longer be sustained since this would involve the further conversion of unreacted reagents. In fact, previous laboratory studies have shown that the yield of the reaction does not change after at least 15 seconds, supporting this theory. ${ }^{16}$

SEM pictures (Fig. 5) taken from a solid sample of the here conducted reaction revealed uniform spherical crystallites of about $100 \mathrm{~nm}$ in diameter sintered together to form larger agglomerates. The calculated average size of coherently scattering domains is thus consistent with the average crystal size found in the SEM analysis.

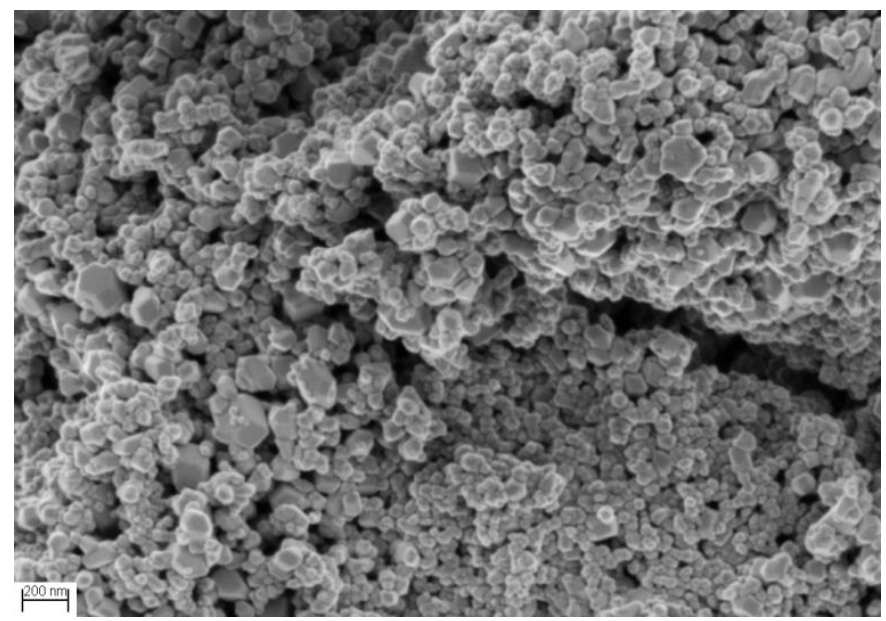

Figure 5: SEM image of ZIF-8 crystals isolated after synthesis

In conclusion, the application of in-situ X-ray diffraction techniques allowed the monitoring of a continuous MOF reaction. Time resolved XRPD shows that ZIF-8 crystallite formation is already completed after $8 \mathrm{sec}$ of reaction time. The ZIF-8 formation process could be followed with high accuracy, revealing that crystal growth occurs simultaneously with nucleation during the first seconds of the reaction.
Kinetic analysis based on the acquired data suggests a diffusion-controlled growth mechanism after the initial nucleation phase, which clearly responds to the flow behavior present in the chosen reactor setup.

It was shown for the first time that continuous reactions can be monitored with the use of high-resolution, highenergy synchrotron XRPD. The advantages of this generally applicable setup include the ability to follow the reaction from the very beginning while increasing the time resolution of the analysis simply by adjusting the flow. Furthermore, various diffraction or spectroscopic methods (i.e. SAXS, Neutron diffraction, IR) can be used for the in-situ observation of the reaction providing a complete understanding of the formation mechanism, paving the way towards process optimization, and giving rise to new opportunities for fast, cheap and repeatable MOF production.

\section{ASSOCIATED CONTENT}

\section{Supporting Information}

The supporting information file contains details concerning the experimental procedures, reactor stage movement and data analysis as well as photos of the experimental setup. This material is available free of charge at http://pubs.acs.org.

\section{AUTHOR INFORMATION}

\section{Corresponding Author}

Angelos Polyzoidis - Fraunhofer Institute for Chemical Technology ICT - Energetic Materials - Joseph-von-Fraunhofer-Str. 7, 76327 Pfinztal, Germany. Phone: +49-721-4640875; e-mail: angelos.polyzoidis@ict.fraunhofer.de.

\section{Notes}

The authors declare no competing financial interests.

\section{REFERENCES}

(1) Ferey, G. Hybrid porous solids: past, present, future. Chem. Soc. rev. 2008, 37, 191-214.

(2) Zhou, H.-C. J.; Kitagawa, S. Metal-organic frameworks (MOFs). Chem. Soc. rev. 2014, 43, 5415-5418.

(3) Stock, N.; Biswas, S. Synthesis of metal-organic frameworks (MOFs): routes to various MOF topologies, morphologies, and composites. Chem. Rev. 2012, 112, 933-969.

(4) Batten, M. P.; Rubio-Martinez, M.; Hadley, T.; Carey, K.-C.; Lim, K.-S.; Polyzos, A.; Hill, M. R. Continuous flow production of metalorganic frameworks. Curr. Opin. Chem. Eng. 2015, 8, 55-59.

(5) Myers, R. M.; Fitzpatrick, D. E.; Turner, R. M.; Ley, S. V. Flow chemistry meets advanced functional materials. Chem. Eur. J. 2014, 20, 12348-12366.

(6) Mazaj, M.; Kaučič, V.; Zabukovec Logar, N. Chemistry of Metalorganic Frameworks Monitored by Advanced X-ray Diffraction and Scattering Techniques. Acta Chim. Slov. 2016, 63, 440-458.

(7) Cravillon, J.; Nayuk, R.; Springer, S.; Feldhoff, A.; Huber, K.; Wiebcke, M. Controlling Zeolitic Imidazolate Framework Nano- and Microcrystal Formation: Insight into Crystal Growth by Time-Resolved In Situ Static Light Scattering. Chem. Mater. 2011, 23, 2130-2141.

(8) Cravillon, J.; Schroder, C. A.; Nayuk, R.; Gummel, J.; Huber, K.; Wiebcke, M. Fast nucleation and growth of ZIF-8 nanocrystals monitored by time-resolved in situ small-angle and wide-angle X-ray scattering. Angew. Chem. Int. ed. 2011, 50, 8067-8071.

(9) Millange, F.; Medina, M. I.; Guillou, N.; Ferey, G.; Golden, K. M.; Walton, R. I. Time-resolved in situ diffraction study of the solvothermal 
crystallization of some prototypical metal-organic frameworks. Angew. Chem. Int. ed. 2010, 49, 763-766.

(10) Ahnfeldt, T.; Moellmer, J.; Guillerm, V.; Staudt, R.; Serre, C.; Stock, N. High-throughput and time-resolved energy-dispersive X-ray diffraction (EDXRD) study of the formation of CAU-1-(OH)2: microwave and conventional heating. Chem. Eur. J. 2011, 17, 6462-6468.

(11) El Osta, R.; Feyand, M.; Stock, N.; Millange, F.; Walton, R. I. Crystallisation Kinetics of Metal Organic Frameworks From in situ TimeResolved X-ray Diffraction. Powder Diffr. 2013, 28, 256-275.

(12) Reinsch, H.; Stock, N. Formation and characterisation of Mn-MIL100. CrystEngComm 2013, 15, 544-550.

(13) Ragon, F.; Horcajada, P.; Chevreau, H.; Hwang, Y. K.; Lee, U.-H.; Miller, S. R.; Devic, T.; Chang, J.-S.; Serre, C. In situ energy-dispersive $\mathrm{X}$-ray diffraction for the synthesis optimization and scale-up of the porous zirconium terephthalate UiO-66. Inorg. Chem. 2014, 53, 2491-2500.

(14) Zahn, G.; Zerner, P.; Lippke, J.; Kempf, F. L.; Lilienthal, S.; Schröder, C. A.; Schneider, A. M.; Behrens, P. Insight into the mechanism of modulated syntheses: In situ synchrotron diffraction studies on the formation of Zr-fumarate MOF. CrystEngComm 2014, 16, 9198-9207.

(15) Friscic, T.; Halasz, I.; Beldon, P. J.; Belenguer, A. M.; Adams, F.; Kimber, S. A. J.; Honkimaki, V.; Dinnebier, R. E. Real-time and in situ monitoring of mechanochemical milling reactions. Nat. Chem. 2013, 5, 66-73.

(16) Polyzoidis, A.; Altenburg, T.; Schwarzer, M.; Loebbecke, S.; Kaskel, S. Continuous microreactor synthesis of ZIF-8 with high spacetime-yield and tunable particle size. Chem. Eng. J. 2016, 283, 971-977.

(17) Crystal structure data CCDC 602542, Cambridge Crystallographic Data Centre, Cambridge, UK.

(18) Herrmann, M.; Kempa, P. B.; Fietzek, H.; Altenburg, T.; Polyzoidis, A.; Piscopo, C. G.; Löbbecke, S. Characterization of MetalOrganic Frameworks Using X-ray Diffraction. Chem-Ing-Tech 2016, 88, 967-970.

(19) Terban, M. W.; Johnson, M.; Di Michiel, M.; Billinge, S. J. L. Detection and characterization of nanoparticles in suspension at low concentrations using the X-ray total scattering pair distribution function technique. Nanoscale 2015, 7, 5480-5487.

(20) Kolmogorov A. N. Statistical theory of phase transformation. Bull. Acad. Sci. USSR. Ser. Math. 1937, 3, 355-359 (in Russian).

(21) Avrami, M. Kinetics of Phase Change. I General Theory. J. Chem. Phys. 1939, 7, 1103-1112.

(22) Avrami, M. Kinetics of Phase Change. II Transformation-Time Relations for Random Distribution of Nuclei. J. Chem. Phys. 1940, 8 , 212-224.

(23) Avrami, M. Granulation, Phase Change, and Microstructure Kinetics of Phase Change. III. J. Chem. Phys. 1941, 9, 177-184.

(24) Pradell, T.; Crespo, D.; Clavaguera, N.; Clavaguera-Mora, M. T. Diffusion controlled grain growth in primary crystallization: Avrami exponents revisited. J. Phys.: Condens. Matter 1998, 10, 3833-3844.

(25) Certificate Standard Reference Material 660a, National Institute of Standards @ Technology, Gaithersburg, MD, 2000. 


\section{Synopsis}

Combination of high-resolution, high energy synchrotron radiation with a specially designed flow reactor allowed for the first time the in-situ observation of a continuous metal-organic framework synthesis from the very first seconds with high accuracy. This technique allowed the optimization of the residence time of the reaction and opened new process windows by identifying the controlling stage of the MOF formation.

Graphic for Table of Contents Only

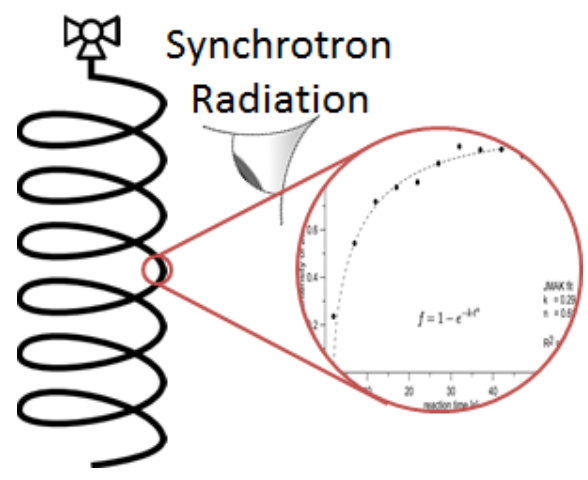

